\title{
Ionic Liquid Technology: A Budding Innovative Podium for the Pharmaceutical Industry
}

\author{
Kapoor $\mathrm{D}^{* 1}$, Vyas $\mathrm{RB}^{1}$ and Dadrwal $\mathrm{D}^{2}$ \\ ${ }^{1}$ Dr. Dayaram Patel Pharmacy College, Surat, Gujarat, India \\ ${ }^{2}$ Sanjivani College of Pharmaceutical Sciences, Rajota, Rajasthan
}

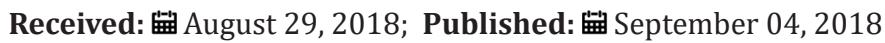

*Corresponding author: Kapoor D, Dr. Dayaram Patel Pharmacy College, Sardar baug, Station Road, Bardoli, Surat, Gujarat, India

\begin{abstract}
Ionic liquids considered to be a reasonably current magical chemical due their unique properties, have a large variety of applications in all areas of the chemical and pharmaceutical industries. The areas of application consist electrolyte in batteries, lubricants, plasticizers, solvents and catalysis in synthesis, matrices for mass spectroscopy, solvents to manufacture nano-materials, extraction, gas absorption agents, etc. Non-volatility and nonflammability are their widespread characteristics giving them a beneficial edge in a variety of applications. Ionic liquids can be considered as green solvents due to their very low vapor pressure and wide range of applications with unique physical and chemical properties. Ionic liquids use for clean and efficient energy, through the development of a broad swath of energy technologies, such as advanced batteries, dye-sensitized solar cells, double layer capacitors, actuators, fuel cells, thermo-cells, and water splitting, essentially related to highly efficient carbon capture and storage technologies and resource efficiency to date. Ionic liquids have emerged as an environmentally friendly substitute to the volatile organic solvents. Being designer solvents, they can be modulated to suit the reaction conditions, therefore earning the name "task specific ionic liquids." They are now finding applications in various fields like catalysis, electrochemistry, spectroscopy, and material science to mention a few.
\end{abstract}

Keywords: Green technology; Chemical industries; Supercritical carbon dioxide; Pharmaceutical application; dye-sensitized solar cells

\section{Introduction}

Chemists and medicinal scientists can significantly diminish the risk to human health and the environment by following all the precious principles of green chemistry. The most uncomplicated and undeviating way to pertain green chemistry in pharmaceuticals is to utilize eco-friendly, non-hazardous, reproducible and competent solvents and catalysts in synthesis of drug molecules, drug intermediates and in researches involving synthetic chemistry. Microwave synthesis is also a significant contrivance of green chemistry by being an energy efficient process. Ionic liquids have been accepted as a new green chemical revolution which excited both the academia and the chemical industries. This innovative chemical group can lessen the utilization of hazardous and polluting organic solvents due to their exclusive characteristics as well as

taking part in various new syntheses. The terms room temperature ionic liquid (RTIL), nonaqueous ionic liquid, molten salt, liquid organic salt and fused salt have all been used to depict these salts in the liquid phase [1]. Ionic liquids (IL) were found to play a special role in the pharmaceutical industry. IL have been defined as compounds that consist quasi-exclusively of ions with a melting point below $100 \circ \mathrm{C}$, and some have gained increasing attention as clean, multifunctional solvents for a variety of applications $[2,3]$. Ionic liquids are known by numerous dissimilar names like neoteric solvents, designer solvents, ionic fluids, and molten salts. Most of the ionic liquids are composed of organic cation and inorganic anions. In order to be liquid at room temperature, the cation should preferably be unsymmetrical; that is, the alkyl groups should be different (Figure 1). 


\section{Ionic Liquids Database- ILThermo}

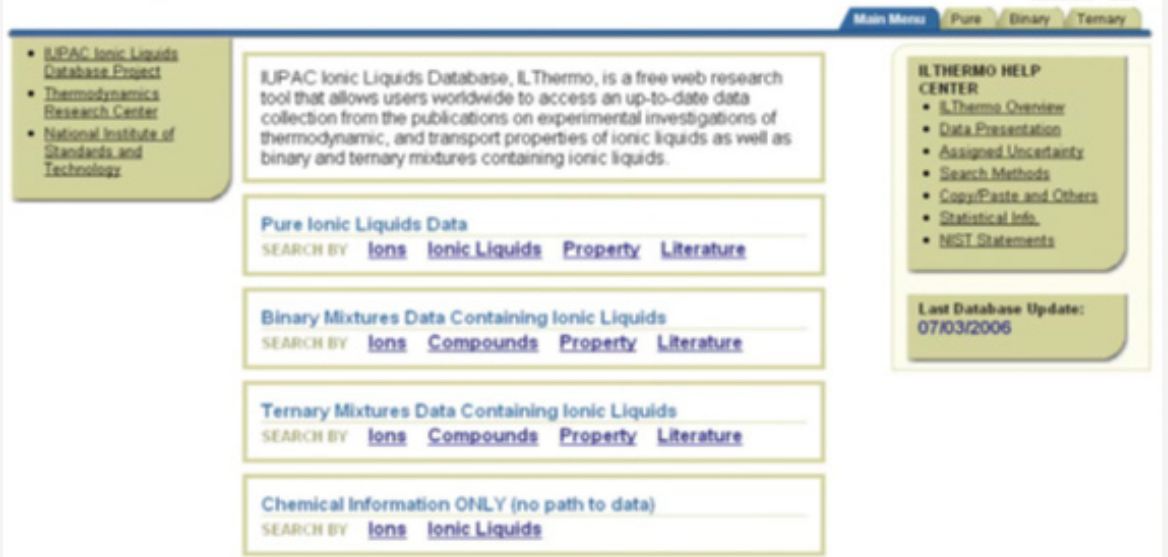

Figure 1: The home page of the IUPAC Ionic Liquids Database.

\section{Properties of Ionic Liquids [4,5]}

I. Made of positively and negatively charged ions, whereas water and organic solvents, such as toluene and dichloromethane, are made of molecules.

II. Structure of ionic liquids is similar to the table salt such as sodium chloride which contains crystals made of positive sodium ions and negative chlorine ions, not molecules.

III. Most of ionic liquids remain liquid at room temperature.

IV. The adopted upper melting temperature limit for the classification as 'IL' is known as $100 \circ \mathrm{C}$ and higher melting ion systems are generally referred as molten salts.

Ionic liquids come in two main categories, namely simple salts (made of a single anion and cation) and binary ionic liquids (salts where equilibrium is involved). For example, [EtNH3] [NO3] is a simple salt whereas mixtures of aluminum (III) chloride and 1,3-dialkylimidazolium chlorides (a binary ionic liquid system) contain several different ionic species and their melting point and properties depend upon the mole fractions of the aluminum (III) chloride and 1,3 dialkylimidazolium chloride present 26. Room-temperature ionic liquids, such as BMIM-PF6 [1-Butyl3-methylimidazolium hexafluorophosphate] have been used as direct replacements for conventional organic solvents in multiphase bioprocess reactions, including liquid-liquid extraction of the antibiotic erythromycin and two-phase biotransformation processes. The addition of organometallic reagents to carbonyl compounds is an important reaction [6] (Figure 2).

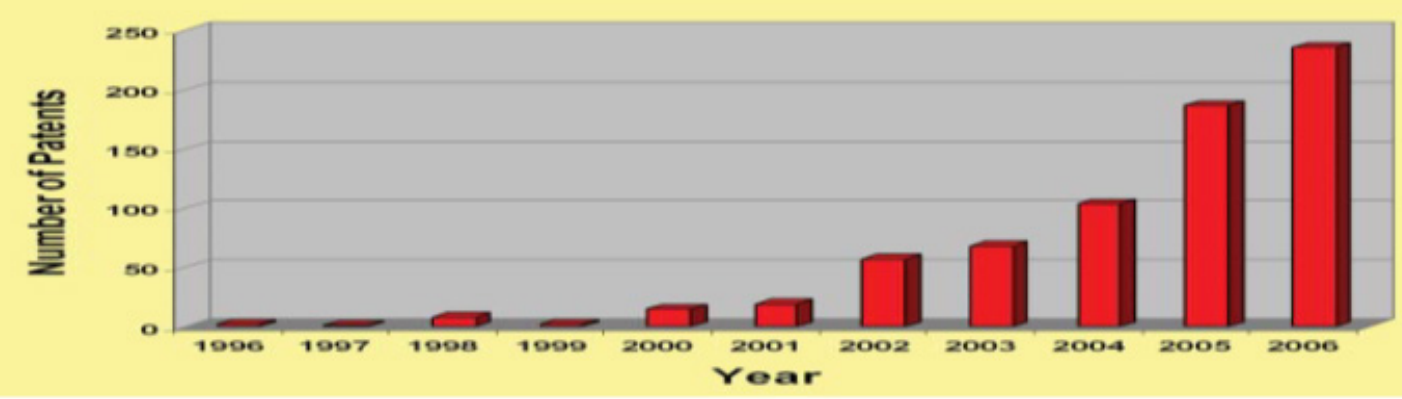

Figure 2: Annual growths of ionic liquid patents, 1996-2006.

\section{Work Done on Ionic Liquids}

a) Room-temperature ionic liquids, such as BMIM-PF6 [1-Butyl-3-methylimidazolium hexafluorophosphate] have been used as direct replacements for conventional organic solvents in multiphase bioprocess reactions, including liquidliquid extraction of the antibiotic erythromycin and two-phase biotransformation processes [6].

b) Numbers of commercially important molecules have been synthesized by Friedel-Crafts acylation reactions in these ionic liquids [7].

c) Ionic liquids with a pyridinium cation bearing an ester side chain moiety can be prepared from either pyridine or nicotinic acids which are biodegradable [8].

d) The addition of organometallic reagents to carbonyl compounds is an important reaction. Formation of homoallylic alcohols, from the addition of allylstannanes to aldehydes in the 
ionic liquids [BMIM-PF4] and BMIM-PF6 have been reported [9].

e) Synthesis of (S)-Naproxen in the ionic liquid BMIM-PF4 has been reported, which is example of asymmetric hydrogenation reactions in ionic medium [10].

f) The very common organic reaction, Friedel-Crafts alkylation proceeds smoothly and efficiently in chloroaluminate (III) ionic liquids [11].

\section{General Properties of Ionic Liquids}

ILs are made of positively and negatively charged ions, whereas water and organic solvents, such as toluene and dichloromethane, are made of molecules. The structure of ILs is similar to the table salt such as sodium chloride which contains crystals made of positive sodium ions and negative chlorine ions, not molecules. While, salts do not melt below $800 \circ \mathrm{C}$, most of ILs remains liquid at room temperature [5].

\section{Advantages: [12]}

a. They have the ability to dissolve many different organic, inorganic and organometallic materials.

b. They are highly polar.

c. It consists of loosely coordinating bulky ions.

d. It does not evaporate since they have very low vapor pressures.

e. They are thermally stable, approximately up to $300 \circ \mathrm{C}$.

f. Most of ionic liquids have a liquid window of up to $200 \circ \mathrm{C}$ which enables wide kinetic control.

g. They have high thermal conductivity and a large electrochemical window.

h. They are immiscible with many organic solvents.

i. They are non-aqueous polar alternatives for phase transfer processes.

j. The solvent properties of ILs can be tuned for a specific application by varying the anion cation combinations.

\section{Solvent Properties of Ionic liquid}

ILs are immiscible with most of the organic solvents, thus they provide a nonaqueous, polar alternative for two-phase systems. Furthermore, ILs which is not miscible with water can be used as immiscible polar phases with water. Although all other conventional solvents evaporate to the atmosphere, ILs does not evaporate and their nonvolatility gives an opportunity to utilize them in high vacuum systems. In most chemical applications, extraction is used for separation since it is an energy efficient technique. Generally, extraction consists of two immiscible phases such as an organic phase and an aqueous phase. Many organic solvents used in extractions are known with their flammable and toxical properties. In order to improve the safety and environmental friendliness of this conventional technique, ILs may be used as ideal substitutes due to their stability, nonvolatility and adjustable miscibility and polarity [13].

\section{Synthesis of Ionic Liquids $[14,15]$}

Ionic liquids come in two main categories, namely, simple salts (made of a single anion and cation) and binary ionic liquids (salts where equilibrium is involved). For example, [EtNH3] [NO3] is a simple salt whereas mixtures of aluminum (III) chloride and 1,3-dialkylimidazolium chlorides (a binary ionic liquid system) contain several different ionic species, and their melting point and properties depend upon the mole fractions of aluminum (III) chloride and 1,3- dialkylimidazolium chloride present. The synthesis of ionic liquids can be described in two steps:

The Formation of the Desired Cation: The desired cation can be synthesized either by the protonation of the amine by an acid or through quaternization reactions of amine with a haloalkane and heating the mixture.

Anion Exchange: Anion exchange reactions can be carried out by treatment of halide salts with Lewis acids to form Lewis acidbased ionic liquids or by anion metathesis.

\section{Active Pharmaceutical Ingredient Based on Ionic Liquids}

\section{Synthesis of Pharmaceutical Salts Ionic Liquids (API-ILs)}

Table 1: Comparison of organic solvents with ionic liquids.

\begin{tabular}{|c|c|c|}
\hline Properties & Organic solvents & Ionic liquids \\
\hline Chirality & Rare & Common and tuneable \\
\hline Vapour pressure & $\begin{array}{l}\text { Obeys the Clausius- } \\
\text { Clapeyron equation }\end{array}$ & $\begin{array}{l}\text { Negligible vapour pressure } \\
\text { under normal conditions }\end{array}$ \\
\hline Flammability & Usually flammable & Usually non-flammable \\
\hline Solvation & Weakly solvating & Strongly solvating \\
\hline Polarity & $\begin{array}{l}\text { Conventional polarity } \\
\text { concepts apply }\end{array}$ & $\begin{array}{l}\text { Polarity concept } \\
\text { questionable }\end{array}$ \\
\hline Tuneability & $\begin{array}{l}\text { Limited range of } \\
\text { solvents }\end{array}$ & $\begin{array}{l}\text { available Virtually } \\
\text { unlimited range means } \\
\text { "designer solvents" }\end{array}$ \\
\hline Cost & Normally & $\begin{array}{c}\text { cheap Typically between } 2 \\
\text { and } 100 \text { times the cost of } \\
\text { organic solvents }\end{array}$ \\
\hline Recyclability & Green imperative & Economic imperative \\
\hline $\begin{array}{l}\text { Number of } \\
\text { solvents. }\end{array}$ & 1000. & $1,000,000$ \\
\hline Applicability & Single function & Multifunction \\
\hline Catalytic ability & Rare & Common and tuneable \\
\hline
\end{tabular}

The physicochemical or biopharmaceutical properties of a drug can be overcome by pairing a basic or acidic drug molecule with 
a counter ion to create a salt-drug. Such salts may offer several advantages compared with the correspondent original neutral formulations in terms of physical properties, such as melting point, crystallinity, hygroscopicity, and dissolution rate, as well as pharmaceutical properties, such as bioavailability, permeability, and drug delivery. In general, the synthetic process is a simple way to modify the properties of a drug with ionizable functional groups in order to overcome undesirable features of the parent drug (94). The selection of ion pairs to form ILs is carried out with candidate ions that have low symmetry and diffuse charge, parameters that also characterize several typical APIs. Even the nitrogen-containing heterocycles, commonly used in ILs today, are frequently found in APIs or API precursors [16, 17] (Table 1) and (Figure 3).

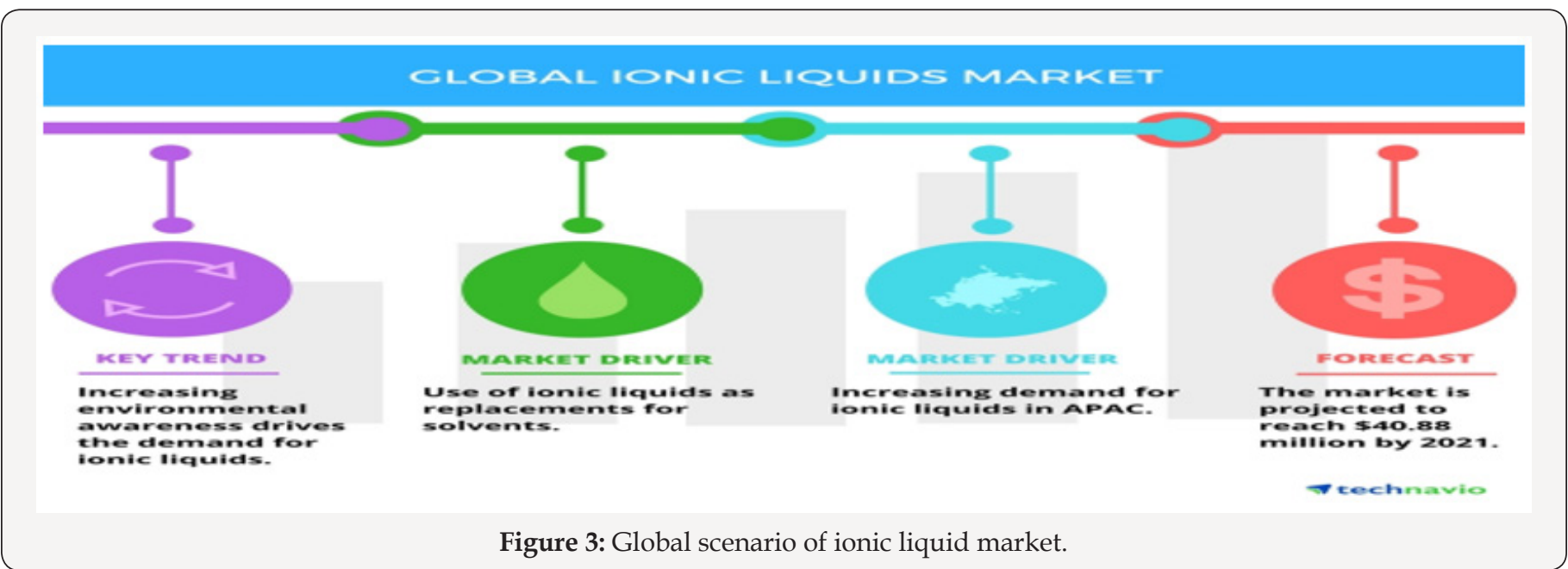

\section{Delivery of drug using ionic Liquids}

Frade \& Afonso published a concise review on the impact of ILs in the environment and humans; they concluded that because IPC81 leukemia cells seemed to be the most vulnerable to ILs within the experimented cell line models, these cells should be used at a first stage in a preliminary screening [18]. Jaitely et al. used a set of ILs with fixed anion and increased the alkyl chain length of the same cation, [C4-8MIM] [PF6], as drug solvents or reservoirs of hydrophobic/hydrophilic, polar/nonpolar drugs where sucrose, penicillin $\mathrm{V}$ potassium, dexamethasone dehydroepiandrosterone, and progesterone were used [19] (Table 2) and (Figure 4).

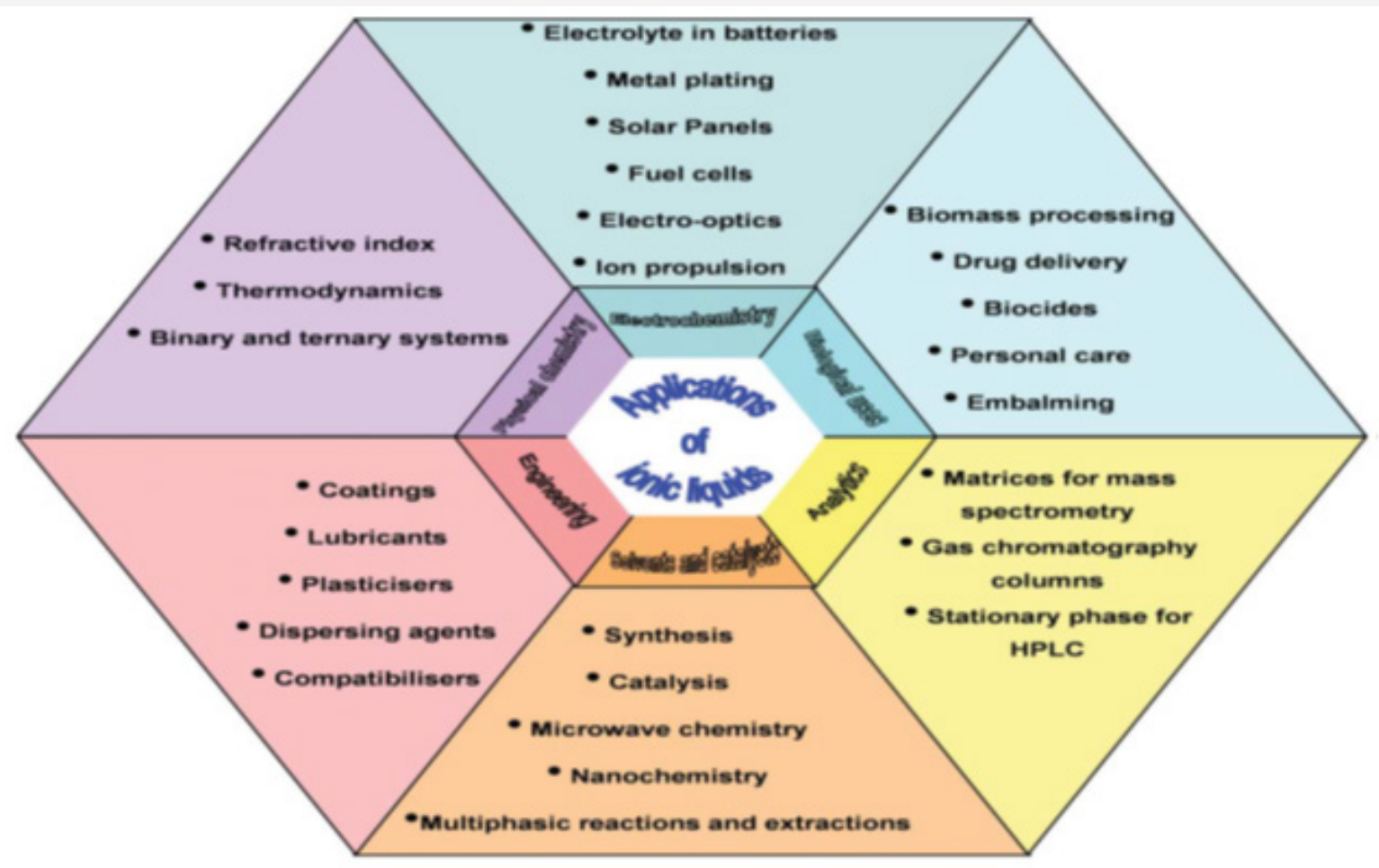

Figure 4: Application of ionic liquids for different sectors. 
Table 2: Some of the major suppliers of ionic liquids.

\begin{tabular}{|c|c|}
\hline Supplier & Website \\
\hline Solchemar & http://www.solchemar.com/ \\
\hline Chemada & http://www.chemada.com/cat0.htm \\
\hline ACROS & $\begin{array}{c}\text { http://www.acros.com/_Rainbow/pdf/AO_ } \\
\text { IonicLiqui_Eur_def.pdf }\end{array}$ \\
\hline Sigma-Aldrich & $\begin{array}{c}\text { http://www.sigmaaldrich.com/catalog/search/ } \\
\text { TablePage/16255866 }\end{array}$ \\
\hline Kanto Chemical Co. & http://www.kanto.co.jp/english/ \\
\hline Nippon Gohsei & $\begin{array}{c}\text { http://www.nichigo.co.jp/english/pro/chemical. } \\
\text { html }\end{array}$ \\
\hline $\begin{array}{l}\text { Merck KGaA/EMD } \\
\text { Chemicals }\end{array}$ & $\begin{array}{l}\text { http://ildb.merck.de/ionicliquids/en/startpage. } \\
\text { htm }\end{array}$ \\
\hline BASF & http://www.basionics.com \\
\hline Cytec & http://www.cytec.com/ \\
\hline SACHEM & $\begin{array}{c}\text { http://www.sacheminc.com/products-and- } \\
\text { services/ionic-liquids.html }\end{array}$ \\
\hline
\end{tabular}

\section{Conclusion}

ILs are receiving more and more attention every day both in academic research and commercial applications and they seem as good replacements for volatile organic solvents. However, there is a discussion about the greenness of the ILs due to their incomplete physical, chemical and toxicological data. most of the researchers will continue to work with this new solvent, the developments of new applications utilizing ILs will increase rapidly and the number of publications will rise exponentially in the future. The present costs of the ILs are quite prohibitive in many probable commercial applications. However, there are hopes that in the near future, the cost/benefit figures of the ILs will bring economic viability to their more common use. The development of pharmaceutical applications using IL-based methodologies requires a deep understanding of ILs both in terms of their macroscopic properties and also at the molecular level, because structural aspects have been shown to play a crucial and unexpected role in a large number of situations. Despite the fact that ILs can no longer be considered a new field, the large diversity of combinations of cations and anions producing novel ILs with new specific properties is astonishing.

\section{References}

1. Sugden S, Wilkins H (1929) The parachor and chemical constitution. Part XII. Fused metals and salts, J Chem Soc pp. 1291-1298.

2. Torchilin VP (2005) Recent advances with liposomes as pharmaceutical carriers. Nat Rev Drug Discov 4(2): 145-160.
3. Seddon KR (1997) Ionic liquids for clean technology. J Chem Technol Biotechnol 68: 351-56.

4. Renner R (2001) Ionic liquids: an industrial cleanup solution, Environ. Sci Technol 35(19): 410A-413A.

5. Yang Q, Dionysiou DD (2004) Photolytic degradation of chlorinated phenols in room temperature ionic liquids. J Photochem Photobiol A Chem 165: 229-240.

6. Cull SG, Holbrey JD, Vargas-MoraV, Seddon KR, Lye GJ (2000) Roomtemperature ionic liquids as replacements for organic solvents in multiphase bioprocess operations. Biotech bioeng 69(2): 227-233.

7. Adams CJ, Earle MJ, Roberts G, Seddon KR (1998) Friedel-Crafts reactions in room temperature ionic liquids. Chem Commun 19: 2097-2098.

8. Jitendra RH, Robert DS, Teresa MG, Peter JS (2008) The design and synthesis of biodegradable pyridinium ionic liquids. Green Chem 10(4): 436-438.

9. Gordon CM, McClusky A (1999) Ionic liquids: a convenient solvent for environmentally friendly allylation reactions with tetraallylstannane. Chem Commun 15: 1431-1432.

10. Monteiro AL, Zinn FK, de Souza RF, Dupont J (1997) Asymmetric hydrogenation of 2-arylacrylic acids catalyzed by immobilized $\mathrm{Ru}-$ BINAP complex in 1-n-butyl-3-methylimidazolium tetrafluoroborate molten salt. Tetrahedron Asymmetry 8(2): 177-179.

11. Boon JA, Levisky JA, Pflug JL, Wilkes JS (1986) Friedel-Crafts reactions in ambient-temperature molten salts. J Org Chem 51(4): 480-483.

12. Zhao H, Xia S, Ma P (2005) Review: use of ionic liquids as green solvents for extractions. J Chem Technol Biotechnol 80: 1089-1096.

13. Kabo GJ, Blokhin AV, Paulechka A, Ya U, Kabo AG, et al. (2004) Thermodynamic properties of 1-butyl-3- methylimidazolium hexafluorophosphate in the condensed state. J Chem Eng Data 49(3): 453-461.

14. Hurley FH, Wier TP (1951) Electrodeposition of metals from fused quaternary ammonium salts. Journal of The Electrochemical Society 98 (5): 203-206.

15. Robinson J, Osteryoung RA (1979) An electrochemical and spectroscopic study of some aromatic hydrocarbons in the room temperature molten salt system aluminum chloride-n-butylpyridinium chloride. Journal of the American Chemical Society 101(2): 323-327.

16. Carter EB, Culver SL, Fox PA, Goode RD, Ntai I, et al. (2004) Sweet success: ionic liquids derived from non-nutritive sweeteners. Chem Commun 6: 630-631.

17. Higasio YS, Shoji T (2001) Heterocyclic compounds such as pyrroles, pyridines, pyrollidins, piperdines, indoles, imidazol and pyrazins. Appl Catal 221(1-2): 197-207.

18. Frade RFM, Afonso CAM (2010) Impact of ionic liquids in environment and humans: an overview. Hum Exp Toxicol 29(12): 1038-1054.

19. Jaitely V, Karatas A, Florence AT (2008) Water-immiscible room temperature ionic liquids (RTILs) as drug reservoirs for controlled release. Int J Pharm 354(1-2): 168-173. 
(C) This work is licensed under Creative

Submission Link:

Submit Article

DOI: 10.32474/DDIPIJ.2018.02.000135

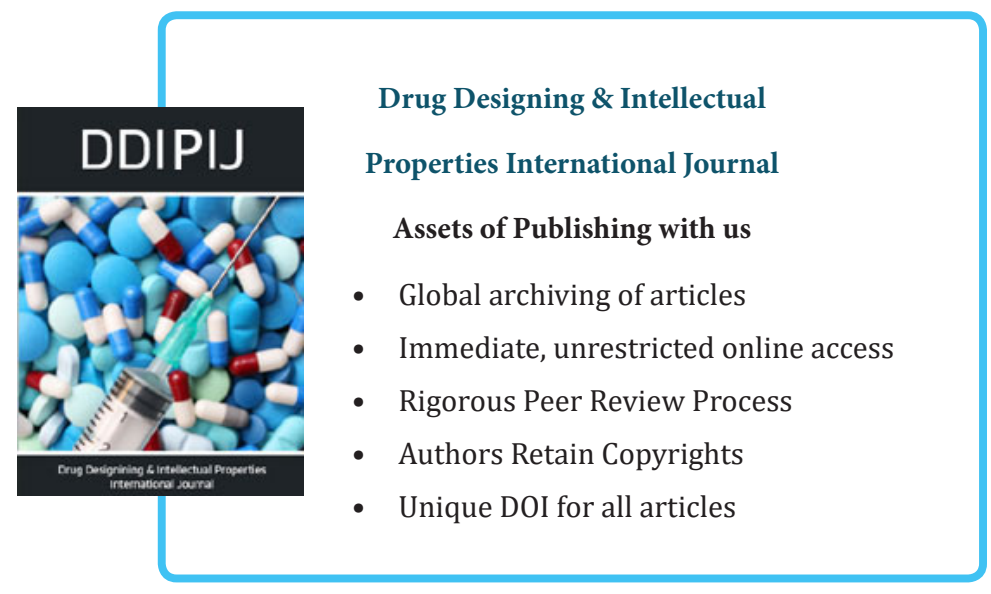

